\title{
GCVS Problems with Flare Stars
}

\author{
Elena V. Kazarovets, Nikolai N. Samus \\ Institute of Astronomy (Russian Academy of Science), 48, Pyatnitskaya Str., \\ Moscow 109017, Russia
}

\section{Introduction}

The astrometric aspect of cataloguing variable stars becomes more and more important. During the past thirty years there has been approximately an threefold increase of the number of known variables; now the rate of new discoveries becomes extremely high, and we may expect a new twofold increase in a couple of years due to discoveries made with space-borne instruments. To avoid confusion, we should continue our policy of naming new variables, and this is impossible without checking identifications with known variable stars, requiring good coordinates and/or finding charts. Operation of automatic telescopes also needs excellent coordinates just for pointing. For all these reasons, we are now working on improving considerably the accuracy standard of variable star coordinates, providing at least reasonably good coordinates for extragalactic variables (GCVS, 4th Edition, Vol. V, now in press, gives coordinates mainly to a second of arc) and variables in globular clusters, identifying variables, including those with rough published positions, with positional catalogues, etc.

Flare stars are very important variables; potentially they are one of the most abundant types of variable stars. Their identification in minimum light may be rather complicated, especially for dense regions of star clusters and associations. Most regretfully, there are many cases of papers on flare stars introducing additional confusion connected with wrong designations, wrong coordinates, missing finding charts. Thus, there appear problems preventing us from incorporating some flare stars in the GCVS. Many definite variables remain in the status of "suspects", being positionally close to known variables, and no finding chart being available either for the earlier known variable or for the new one. Sometimes we do include such a "pretender" into the GCVS, and years after a finding chart or good coordinates appear, and it turns out that we have two entries for the same star (see, for instance, numerous cases of duplicated names for flare stars in Taurus in the GCVS Vol. III).

We shall present here some typical examples of confusion. We ask the authors of papers we are going to quote to excuse us for using these particular papers as examples. 
The original presentation of this paper at the Colloquium included demonstration of tables with originally published and corrected coordinates for stars mentioned in Sections 3 and 4 . These tables (containing coordinates to higher accuracy than those given in the GCVS or Name-lists) may be obtained from the authors upon request. Corrected coordinates are presented in Name-lists of variable stars Nos. 71 and 72 and in the 5 th Volume of the GCVS (in press).

\section{An example of confused designations, coupled with absence of a finding chart}

Melikian \& Della Valle (1986) announced the discovery at Byurakan of two flare stars in Orion, B31 $\left(5^{\mathrm{h}} 23^{\mathrm{m}} \cdot 2,-6^{\circ} 58 ! 5,1900.0\right)$ and B32 $\left(5^{\mathrm{h}} 31^{\mathrm{m}} 0,-6^{\circ} 11^{\prime} 0\right.$, 1900.0). The first of them was later designated V1194 Ori. The discoverers did not publish finding charts. The catalogue of Natsvlishvili (1991) of flare stars in Orion presents photographic charts for B31 and B32; however, this is not the same $\mathrm{B} 32$ but the star at $5^{\mathrm{h}} 33^{\mathrm{m}} 06 \mathrm{~s},-4^{\circ} 18.4(1900.0)$, first discovered by Sugano (Kosai 1983) as an FU Ori-type object and named B32, for its flare activity discovered at Byurakan, by Mirzoyan et al. (1988). Natsvishvili (1992, private communication) now suggests to designate Sugano's star B32A; it is also known as V1143 Ori. The lack of a finding chart for the "first" B32, a star in a field rich in variables, combined with rather low accuracy of its published coordinates, does not permit us to include this star in the GCVS.

\section{An example of very inaccurate coordinates and a finding chart not accessible for the majority of investigators}

Kelemen (1987) announced his discovery of a number of flare stars in the Pleiades. Our discussions with several experts in the field show that people are not able to find many of these stars and express doubt on the reality of these stars at all. Upon our request, Dr. Kelemen sent to Moscow a photographic finding chart of his stars. Using it, we have been able to identify Kelemen's variables. However, the published coordinates for many of them are completely wrong. Among Kelemen's flare stars from the 71st Name-list of variable stars (Kazarovets et al. 1993), the coordinates published by Kelemen were correct only for V1036 Tau, the errors reach $50^{s}$ of time in right ascension and almost $13^{\prime}$ in declination. This is probably due to misidentifications of some reference stars during positional measurements. Of 28 identifications with stars from Haro et al. (1982), suggested by Kelemen (1987), we confirm only one. Surely no one will ever find these stars without a chart.

Accurate coordinates for almost 550 flare stars in Pleiades were measured by Kazarovets (1993); this paper also discusses several cases of identification being impossible because of lack of finding charts. 


\section{An example of "accurate" coordinates actually being rather rough, but in the presence of a good chart}

Chavira et al. (1992) discovered a number of variable stars in the Orion nebula region and published their rather rough positions for equinox 1900.0. Then, Parsamian et al. (1993) published a good field photograph for these variables. The latter paper contains coordinates of the variables for the equinox 1950.0 to 0.01 in right ascension and to $0 . \prime 01$ in declination. However, these coordinates actually are mostly quite rough. In the recently finished 72nd Name-list of variable stars (Kazarovets \& Samus 1995), we present accurate coordinates, either taken from the GSC or determined by S. Antipin using reference stars from the GSC; the identifications with the published photograph met no problem. It seems that practically in all cases (notable exceptions are V1263 Ori and V1297 Ori, where it is difficult to find any explanation) rough coordinates from Chavira et al. 1992 were simply transformed to the new equinox, using not the best algorithm, and more digits were retained in the result than it was permitted by the original data. This could be a rather serious problem, if there were no finding chart.

\section{Conclusions}

These examples clearly show the importance of good finding charts for flare star identifications. Though continuously emphasized by IAU Comm. 27, the situation does not improve noticeably. Flare stars without finding charts constitute $36 \%$ of all flare stars in the Name-lists Nos. 67-71, compared to $7 \%$ of RR Lyraes without finding charts in the same lists. We expect a drastical increase of the number of known variables due to discoveries with space-borne telescopes. This will make identifications and positional work the most laborious and important part of compiling variable star catalogues. Kind cooperation of flare star discoverers is quite necessary. We ask our colleagues to take some more effort and find improved positions, publish finding charts or, at least, GSC identifications. This will spare much more effort in subsequent work.

Acknowledgments: Thanks are due to Mr. S. Antipin for GSC identifications and improved positions for new flare stars in Orion.

\section{References}

Chavira E., González G., Escamilla C., Parsamian E., 1992, IBVS 3764

Haro G., Chavira E., González G., 1982, Bull. Inst. Tonanzintla 3, No. 1

Kazarovets E.V., 1993, Peremennye Zvezdy (Variable Stars), 23, No. 3, 141

Kazarovets E.V., Samus N.N., 1995, IBVS 4140

Kazarovets E.V., Samus N.N., Goranskij V.P., 1993, IBVS 3840

Kelemen J., 1987, IBVS 3103

Kosai H., 1983, IAU Circular No. 3763

Melikian N.D., Della Valle M., 1986, IBVS 2929

Mirzoyan L.V., Melikian N.D., Natsvlishvili R.Sh., 1988, Astrofizika 28, No. 3, 540

Natsvlishvili R.Sh., 1991, Astrofizika 34, No. 1, 107

Parsamian E.S., Chavira E., González G., 1993, Rev. Mex. Astr. Astrofis. 25, No. 2, 71 